\title{
Tadpole-Shaped POSS-Based Copolymers and the Aggregation Behavior at Air/Water Interface
}

\author{
Lin Zhu, ${ }^{1,2,3}$ Fang Chen $\mathbb{D}^{1,2,3}$ Xiaoyan Ma, ${ }^{1,2,3}$ Xiu Qiang, ${ }^{2,3}$ Zhiguang Li, ${ }^{2,3}$ \\ Chen Dong, ${ }^{2,3}$ and Duyang Zang ${ }^{3}$ \\ ${ }^{1}$ Research \& Development Institute, Northwestern Polytechnical University, Shenzhen, China \\ ${ }^{2}$ Key Laboratory of Polymer Science and Technology, Shaanxi Province, School of Science, Northwestern Polytechnical University, \\ Xian 710129, China \\ ${ }^{3}$ Key Laboratory of Space Applied Physics and Chemistry, Ministry of Education, School of Science, Northwestern Polytechnical \\ University, Xian 710072, China
}

Correspondence should be addressed to Fang Chen; chenfang820811@nwpu.edu.cn

Received 21 August 2017; Accepted 18 October 2017; Published 1 January 2018

Academic Editor: Da-Ren Hang

Copyright (C) 2018 Lin Zhu et al. This is an open access article distributed under the Creative Commons Attribution License, which permits unrestricted use, distribution, and reproduction in any medium, provided the original work is properly cited.

\begin{abstract}
The aggregation behavior of three tadpole-shaped Polyhedral oligomeric silsesquioxane (POSS) based block copolymers using different blocks poly(methyl methacrylate) (PMMA) and poly(trifluoroethyl methacrylate) (PTFEMA) with different block sequence and ratio (POSS-PTFEMA 161 -b-PMMA 236 , POSS-PMMA ${ }_{277}-b-$ PTFEMA $_{130}$, and POSS-PMMA $466-b-$ PTFEMA $_{172}$ ) was investigated on the air-water interface. The interfacial rheology of three block copolymers was studied by surface pressure isotherm, compression modulus measurements, and compression and expansion hysteresis analysis on the Langmuir trough. The block sequence and ratio play a great role in self-assembly behavior at the interface. Based on surface pressure isotherm analysis, a thin film with low elasticity was achieved for the POSS-PTFEMA ${ }_{161}-\mathrm{b}-\mathrm{PMMA}_{236}$. Moreover, for the block copolymer with same segment sequence (POSS-PMMA $-b$-PTFEMA), the thin film compression capability is increased with increasing the PMMA ratio. The morphology of the deposited LB thin film was illustrated by atomic force microscopy (AFM) and X-ray photoelectron spectroscopy (XPS). We observed that a thin film was composed by crater-shaped quasi-2D micelles for POSS-PTFEMA- $b$-PMMA, while it was proved that only flaky texture was observed for both POSS-PMMA $277-b-$ PTFEMA $_{130}$ and POSS-PMMA $466-b$-PTFEMA 172 . The thickness and area of flaky aggregates were greatly related to PMMA ratio. The different interface self-assembly structure evolution was proposed based on the interfacial rheology and thin film morphology studies.
\end{abstract}

\section{Introduction}

Polyhedral oligomeric silsesquioxane (POSS), with the welldefined cage-like silica cubic nanoscale, has received considerable attention. POSS may be referred to as a silica nanoparticle consisting of a silica cage core, as well as other organic functional groups attached to the corners of the cage, which consist of hybrid organic-inorganic composition [13]. The cage-like core is a typical 3D hollow structure with a $1.5 \mathrm{~nm}$ scale, which provides excellent properties such as mechanical strength and dielectrical property. Moreover, the design ability and compatibility of POSS are improved by the higher reactivity of organic functional or reactive groups. Hence, POSS-based hybrid materials are widely applied in packing, catalytic, thermal, and oxidative resistance, and a variety of hybrid nanocomposites [4-6].

Since reported by Seo and coworkers [7], interfacial selfassembly of POSS-based small molecules or polymers has attracted great interest during the past few decades. Subsequently, a variety of POSS-based small molecules or polymers were synthesized and the different self-assembly behavior was achieved as well [8-12]. Wamke et al. [13] successfully synthesized two open-cage TSiO-POSS and DSiB-POSS with short alkyl chains and compared the interfacial property of Langmuir monolayer. Jiang et al. [14] prepared a POSS-based amphiphilic supramolecule from the host-guest inclusion complexation between a mono adamantane-functionalized 
TABLE 1: Characteristics of copolymers.

\begin{tabular}{|c|c|c|c|c|}
\hline \multirow{2}{*}{ Sample } & \multicolumn{3}{|c|}{ Mean molecular weight (g/mol) } & \multirow{2}{*}{ PDI } \\
\hline & Total & PMMA & PTFEMA & \\
\hline POSS-PTFEMA $_{161}-b-$ PMMA $_{236}(\mathbf{P 1})$ & 52100 & 23600 & 27000 & 1.94 \\
\hline POSS-PMMA $_{277}-b$-PTFEMA ${ }_{130}$ (P2) & 50500 & 27700 & 21800 & 1.47 \\
\hline POSS-PMMA $_{466}-b$-PTFEMA 172 (P3) & 76500 & 46600 & 28900 & 1.92 \\
\hline
\end{tabular}

POSS (AD-POSS) and a $\beta$-cyclodextrin oligomer (P $(\beta$-CD)). Hollow nanospheres supramolecular hybrids with stable thick wall were obtained at the water/toluene interface. Gunawidjaja et al. [15] synthesized two series of organicfunctionalized branched POSS with various hydrophobichydrophilic terminal groups probing different membrane assembly behavior among bulk state, monolayer, and multilayer films. Zhang et al. [16] designed and synthesized a new structure of amphiphilic copolymer brushes with POSS as side chains and their self-assembly behavior in aqueous media was also studied. The results showed that these alternating copolymer brushes could form spherical aggregates in water. Therefore, POSS is regarded as significant assembled materials at air/water interface since it can exhibit rich aggregated morphology and improve thermal and mechanical properties of thin films.

Diblock copolymers are a material of choice for achieving nanostructures based on phase separation between dissimilar blocks. And diblock copolymer monolayers with nanopattern and recognized assembling mechanism through interface self-assembly are increasingly studied [17-21]. Moreover, by changing structure and external condition such as temperature, irradiation, and pressure or by adding other substances, the tunable nanostructures with different size and shape could be achieved [22-28]. In particular, weakly amphiphilic diblock copolymers, containing both hydrophobic block and surface active but water insoluble block, are attractive for researchers due to excellent stability on the water surface, and the intermolecular interactions mainly drive the assembly of copolymers [29-32]. Therefore, the molecule structure such as chain segments, block ratio, and block sequence becomes a dominant influencing factor on aggregation behavior. Seo et al. [7, 22, 29, 33, 34] synthesized a series of Polystyrene-b-poly(methyl methacrylate) (PS- $b$ PMMA) diblock copolymers and found that these kind of copolymers could form ordered quasi-2D surface micelles at air/water interface. Moreover, the factors such as chemical constitutions, molecule weight, and block ratio could strongly affect the aggregation behavior and interfacial viscoelasticity. Li Destri et al. [30] further reported the viscoelastic behavior of three PS- $b$-PMMA copolymers with different block length ratio, proving that the viscoelastic behavior is changing from a predominantly elastic to a viscoelastic one before and after conformation transition. Chen et al. [35] found that the aggregation behavior of X-shaped block copolymers at air/water interface can be changed by the variation of the block sequence or temperature. Fluorinated copolymers, especially the fluorinated acrylate copolymers, are excellent choice for design weakly amphiphilic diblock copolymers due to hydrophobicity and high surface activity at air/water interface [8, 36-41]. Zhu et al. [42] examined the formation of various PVDF Langmuir films. They found that the spreading solvents play a key role in the properties of PVDF Langmuir films at the air-water interface. Fluorinated materials are capable of self-migration to the surface, resulting in significant effect for surface properties [43-45].

In recent years, there are some very interesting works about combining the functional nanoparticles and copolymers in homogeneous system and constructed nanopatterned thin films. Although self-assembly of block copolymers and inorganic NPs complex have been studied in film form, few experiments of this sort have been conducted at the monolayer level on a Langmuir trough at air/water interface [40, 46, 47]. The copolymer's structure and nanoparticles' properties could both influence the interfacial aggregation behavior of thin films. Importantly, the resulting interaction between copolymers and nanoparticles, such as the location of nanoparticles within polymer matrices, the sequence they combined with each other, is considered to be of the predominant influencing factors in assemble process and received a lot attention [48-51].

Inspired by the above researches, we designed and synthesized three tadpole-shaped POSS-based PMMA- $b$-PTFEMA weakly diblock copolymers using step ATRP technique. Then the air/water interfacial aggregation behavior and surface morphology of all three copolymers were investigated using Langmuir-Blodgett technique. The result presented that all copolymers could form typical Langmuir thin films at air/water interface containing interesting patterns at air/water interface. The block sequence and ratio of PTFEMA- $b$ PMMA could affect the self-assembly behavior of copolymers in different way and reflected in the different properties. Meanwhile, these two factors both strongly affect the surface morphology of film.

\section{Materials and Methods}

2.1. Materials. The tadpole-shaped POSS-based copolymers used in this study were synthesized via atom transfer radical polymerization (ATRP) in our laboratory which is detailedly expressed in SI. The monofunctional POSS (POSS-Cl) was synthesized through vertex-cap measurement and used as initiator of ATRP. The structural characterizations of all three copolymers were verified by ${ }^{1} \mathrm{H}$ NMR spectra and GPC, as exhibited in Supporting Information. The structural characteristics of all three copolymers were shown Table 1 as a result.

2.2. Langmuir Films and Langmuir-Blodgett Films. The dilute solutions of all three copolymers were obtained via copolymers dissolved in chloroform. Concentration of $0.3 \mathrm{mg} / \mathrm{mL}$ 


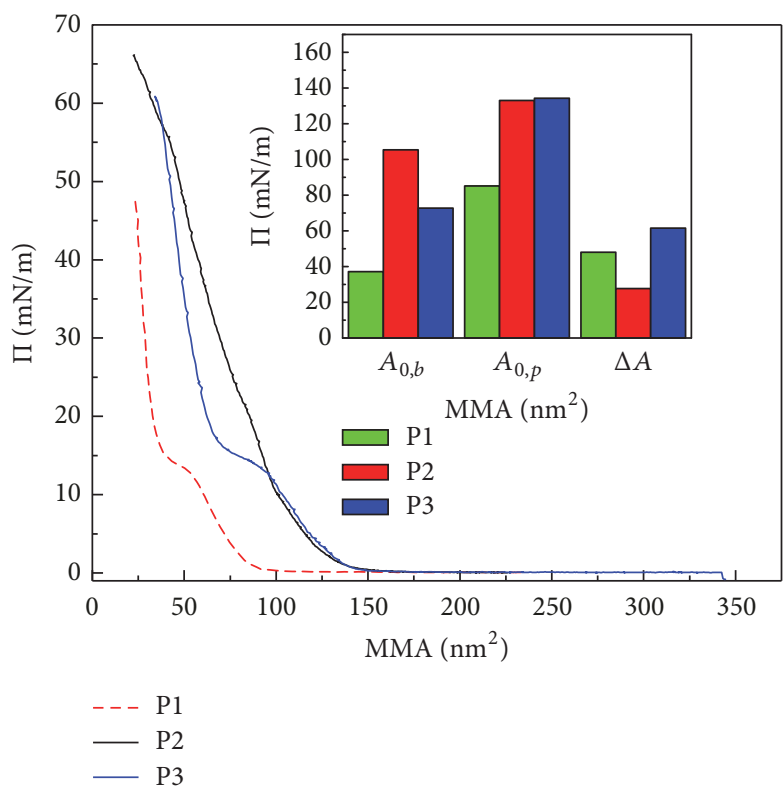

(a)

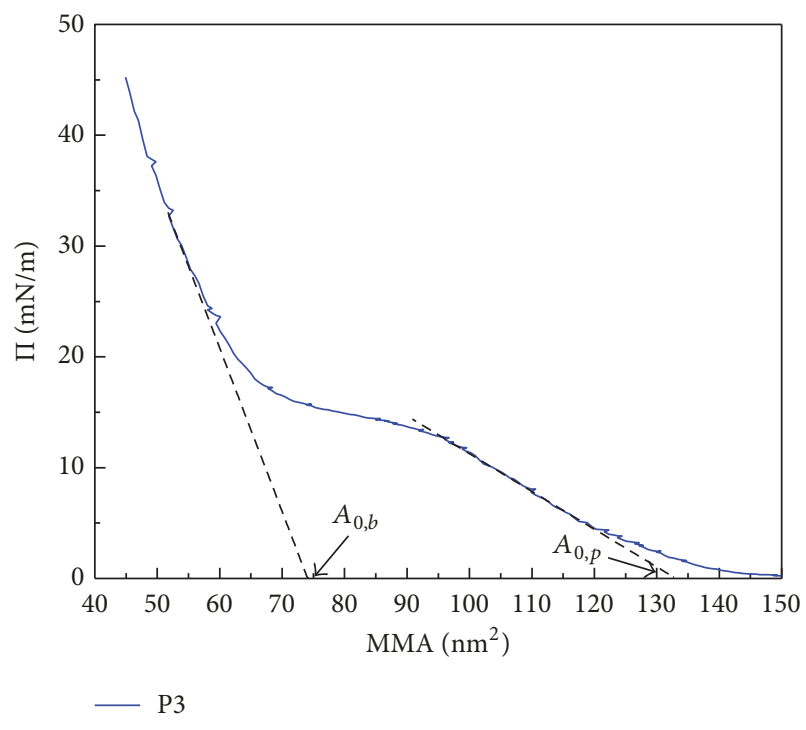

(b)

Figure 1: (a). $\pi$-MMA isotherm of all three copolymers at the air-water interface upon compression. The graph in top right corner is histogram of the characteristic limiting mean molecule area of all three copolymers for region 2 and region 3. (b). The calculation method of $A_{0, p}$ and $A_{0, b}$.

and copolymer additive weight of $0.02 \mathrm{mg}$ were employed for all dilute solutions. In order to make sure of the complete dissolution of copolymers, solutions were put in room temperature for at least $12 \mathrm{~h}$ before preparing Langmuir films. The Langmuir films and Langmuir-Blodgett Films were accomplished using the Langmuir trough with a surface of $200 \times 310 \mathrm{~mm}^{2}$ (JML04C3, power each Ltd., China) and equipped with surface pressure measurement, LangmuirBlodgett film deposition. The subphase water was purified using an ultrapure water system. To prepare the Langmuir film, solutions were deposited drop by drop in a grid pattern on ultrapure water surface. Thirty minutes were allowed to wait for evaporating the solvent before compression. The surface was symmetrically compressed by barriers advancing at a speed of $9.71 \mathrm{~mm} / \mathrm{min}$. The glass substrate was immersed under the water before the polymer solution spread onto the water surface. It has been $30 \mathrm{~min}$ after chloroform volatilization that the submerged substrate was lifted vertically with a speed of $4.5 \mathrm{~mm} / \mathrm{min}$. All procedures are applied under $25^{\circ} \mathrm{C}$.

2.3. Atomic Force Microscopy. All the LB films samples were scanned out with AFM (SPM-9700, Shimadzu, Japan) in tapping mode using silicon probes (FMR-10, Nanoworld, USA). Silicon cantilevers (MikroMasch) with a characteristic resonance frequency of $75 \mathrm{kHz}$ and a force constant of $2.8 \mathrm{~N} / \mathrm{m}$ were used. Tips had a radius of curvature of $10 \mathrm{~nm}$.

2.4. X-Ray Photoelectron Spectroscopy. Structural properties of the LB films were characterized by XPS (K-Alpha, East Grinstead, England) with monochromatic Al Ka X-ray excitation $\left(h_{v}=1486.6 \mathrm{eV}\right)$. The spectrum was analyzed by means of XPS-peak-differentiation-imitating.

\section{Results and Discussion}

3.1. Surface Pressure-Mean Molecular Area ( $\pi-M M A)$ Isotherms. After spreading polymer solution at the air/water interface in Langmuir trough, the surface pressure of copolymers is plotted as function of mean molecular area ( $\pi$-MMA). By studying the $\pi$ as a function of MMA, the aggregation behavior of copolymer at different surface pressure could be specifically revealed. Figure 1(a) showed the $\pi$-MMA isotherm obtained by all three copolymer solutions spreading on Langmuir trough and laterally compressed. The considerable large occupied molecular area is observed by all Langmuir films under low surface pressure in Region 1. Actually the surface pressure is close to $0 \mathrm{mN} / \mathrm{m}$ like pure water. There is no contact and interaction between each molecule at that region. Tiny difference of three copolymer films aggregation in region 1 is revealed, caused by large distance among molecules and similar molecular structures. Moreover, by Maestro et al.s study $[52,53]$, the ester groups of PMMA and PTFEMA blocks tend to point downward the water phase. We supposed that the two blocks are partially immersed in the subphase (water). Meanwhile, the hydrophobic POSS float on the subphase to avoid unfavorable interaction with water. The initial compression causes only a decrease of distance among molecules.

In region 2 shown in Figure 1(a), all the isotherms present steeper slope than region 1 , which indicate that the surface 
pressure starts to increase with the decrease of MMA. P2 and P3 showed highly similarity of compression curve in region 2. But different behavior is detected between P1 and P2(P3) with respect to difference in slope of region 2 . Besides, The limiting MMAs of the pancake and brush conformations $\left(A_{0, p}\right.$ and $A_{0, b}$, resp.) could be quantified by extrapolating tangents to the inflection points of the $\pi$-MMA curves to a surface pressure of zero in Figure 1(a). The difference of limiting MMA ( $\left.\triangle A_{\text {pseudoplateau }}\right)$ is an effective quantitative approach to determine the compressibility and a direct evidence of different self-assembly behavior during compression.

$A_{0, p}$ could provide information of the initial molecular spreading conformation of copolymer in region 2 , which mainly depends on the block sequence and molecular weight. P1 shows a relative smaller $A_{0, p}$ due to the more interaction between terminal PMMA groups compared to P2. And P3 presents the biggest $A_{0, p}$ among the three copolymers mainly due to the highest molecular weight and longer PMMA block length. During compression, the phase-transition limiting area $A_{0, b}$ will represent self-assembly behavior and rearrangement based on the difference in intermolecular interaction. It is shown that $\mathrm{P} 1$ still presents lowest value of $A_{0, b}$. When PMMA lies between POSS and PTFEMA blocks (P2 \& P3), smaller $A_{0, b}$ is observed for P3 due to the higher compressibility.

It is reasonable to propose $\mathrm{P} 1$ with the higher compressibility by comparing $A_{0, b}$ in region 2 of $\mathrm{P} 1$ and $\mathrm{P} 2$, due to the direct bonding between PTFEMA blocks and POSS. Moreover, the block copolymer with longer PMMA length (P3) exhibits higher compressibility in a more compacted state (region 3) while the block sequence is the same. $\Delta A_{\text {pseudoplateau }}$ further describes the compressibility of the three copolymer films from region 2 to region 3 . P3 presents the highest $\triangle A_{\text {pseudoplateau value attribute to the longest }}$ PMMA ratio. It is worth noting that although P1 could easily aggregate, some determined self-assembly structure might be formed and the compressibility is hard to change during the compression.

3.2. Compression Modulus Isotherms. The compression modulus represents the interfacial rheology of the formed Langmuir films at air/water interface; it can be obtained from the slope of $\pi-\Gamma$ or $\pi-A$ :

$$
E=\Gamma\left(\frac{\partial \pi}{\partial \Gamma}\right)
$$

The $E-\Gamma$ plots will provide very important information such as the thin film rigidity and the ability of storing elastic energy. In general, high density of packed molecules will be determined by showing a high $E$ value in Langmuir film.

As shown in Figure 2, one can clearly determine the difference in thin film elasticity. After comparing $E$ of P1 and P2, P1 thin film presents lower elasticity and higher compressibility due to the sequence of PMMA block. While the PMMA is the terminal block, less repulsion is detected between the nearby molecules during compressing. The P3 shows the maximum $E$ value; it is probable because interaction between PTFEMA adjacent molecular is stronger than

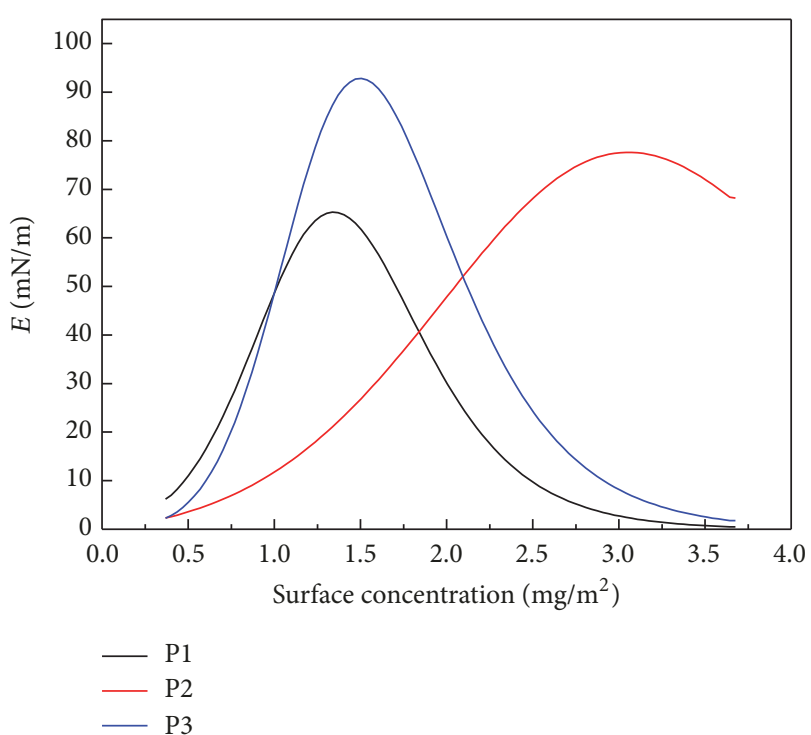

FIGURE 2: E-surface concentration plots of three block copolymers.

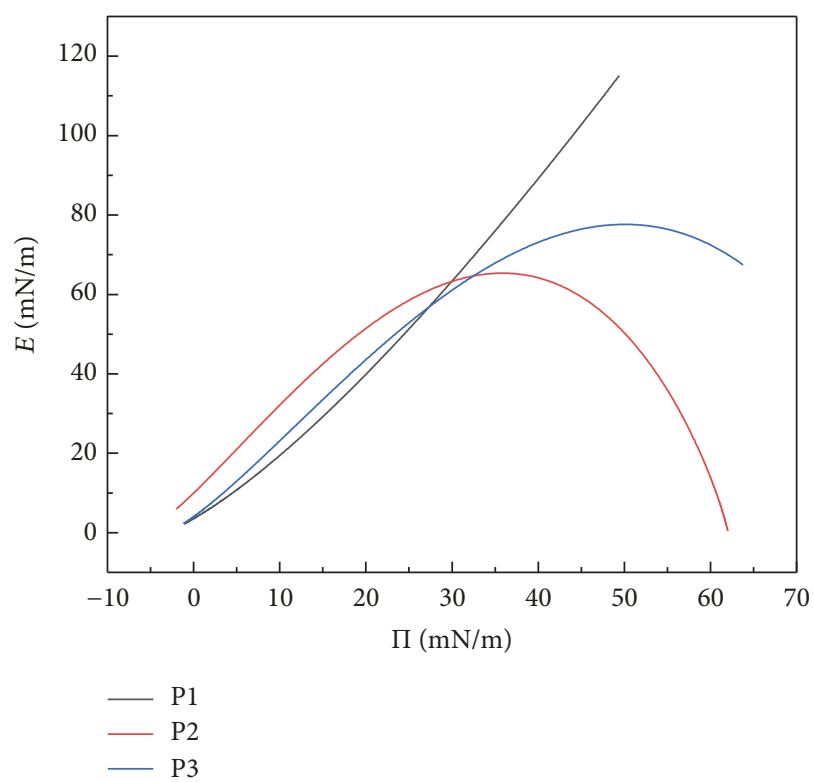

FIgURE 3: Compression modulus as a function of surface pressure of three copolymers.

that of P1. Meanwhile, the thin film is storing higher elastic energy attributed rearrangement during the compression for the P3 with longer PMMA block.

The surface pressure is assumed to be proportional to the surface density following a scaling law $\pi-\Gamma^{y}$ with a scaling exponent $y=2 v /(2 v-1)$ [54]. With the combination of the equilibrium compression modulus is as follows: $E=y \Gamma$. Three different regions are shown in the fitted curves, including dilute, semidilute, and concentrated. In the semidilute region, the compression modulus increased linearly with the increasing of surface pressure with a linear relationship. The $E-\Gamma$ plots are shown in Figure 3, and $y$ values are obtained by 


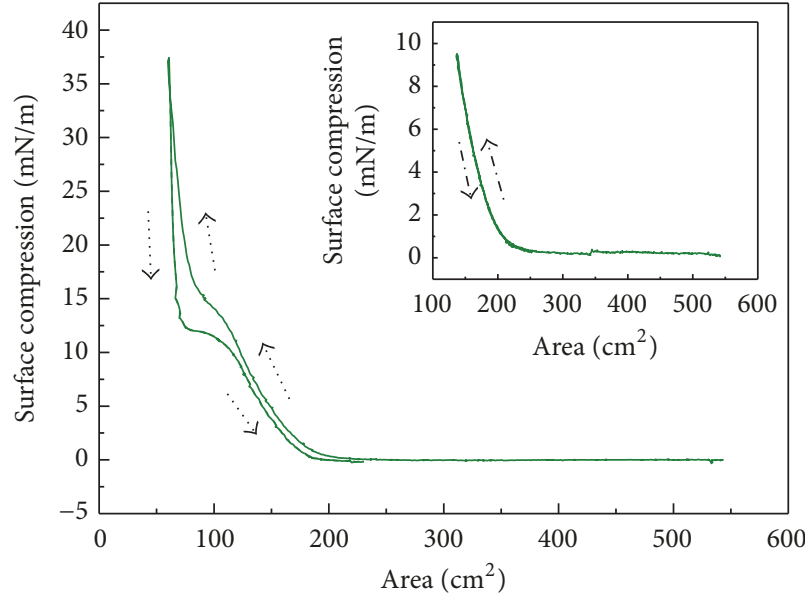

(a)

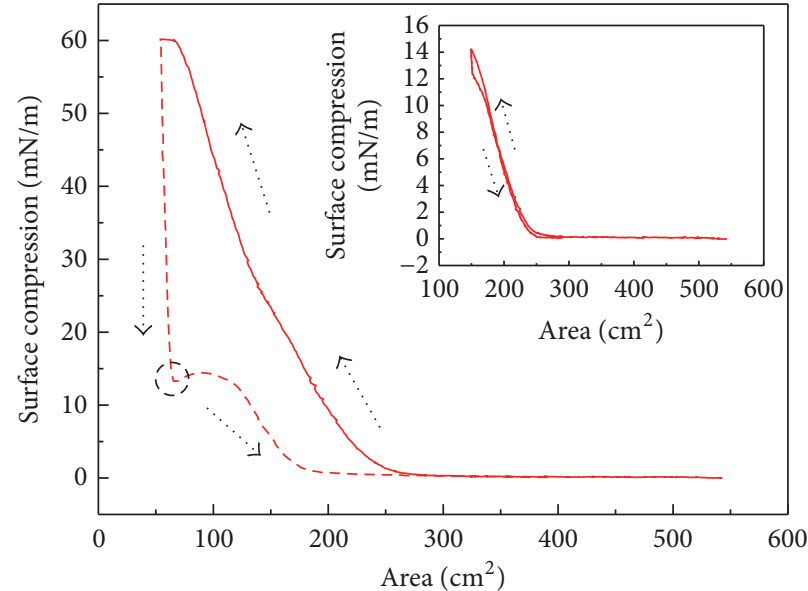

(b)

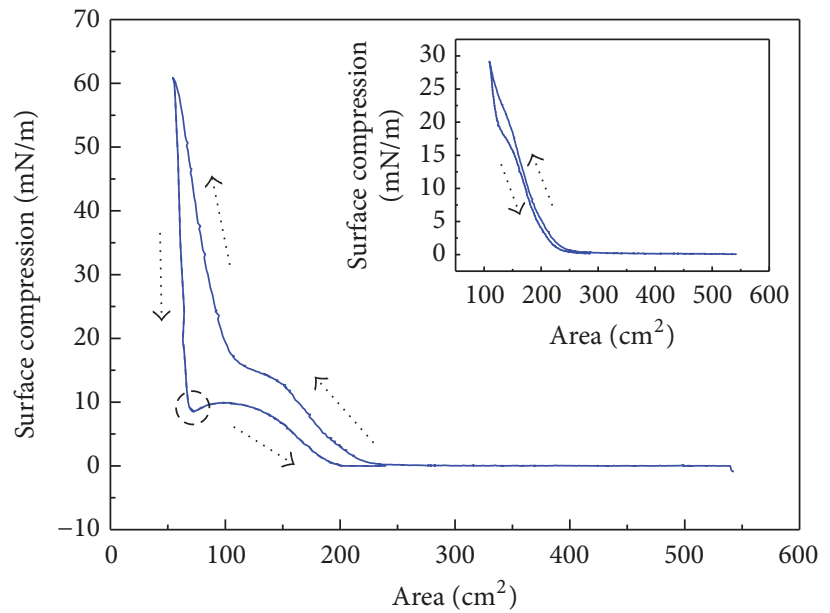

(c)

FIgURE 4: The hysteresis cycles of (a) P1, (b) P2, and (c) P3. The main graphs are hysteresis cycles observed at region 3. Each embedded graph at the top right corner of main graph is hysteresis cycles at region 2.

fitting the slope of semidilute region of $E-\Gamma$, while the $v_{1}, v_{2}$, and $v_{3}$ are calculated as $0.5,0.55$, and 0.55 , respectively. It is clear that the interface exhibits as a poor solvent for the three copolymers.

Hysteresis. To reveal the viscoelastic property of Langmuir films, the hysteresis cycles of P1, P2, and P3 formed thin films under pancake (inset images) and brush regions are, respectively, shown in Figures 4(a), 4(b), and 4(c). From the hysteresis cycles from three copolymers under pancake region (before liquid-solid transition), we could figure out the weak interaction between the molecules since no apparent hysteresis behavior is determined. However, different hysteresis behavior is obtained for all three copolymers in brush region during compression and expansion processes. It is worth noting that all the three copolymer present hysteresis loop in the brush region. In particular, surface pressures of both $\mathrm{P} 2$ and $\mathrm{P} 3$ drop to a certain area during the expansion process; there is an apparently minimum surface pressure observed and highlighted by dashed circles. During expansion, the surface pressure is always lower than that of the compression at the same MMA. Based on Seo et al's arguments, the minimum surface pressure represents structural rearrangement under a slow compression and expansion rate due to higher compressibility of PMMA segment. In our cases, $\mathrm{P} 2$ and $\mathrm{P} 3$ showed a higher elasticity because of larger repulsion of terminal PTFEMA blocks. At the same time, the highest $E$ of P3 are mainly attributed to higher entanglement among longer PMMA block. Among the hysteresis cycles of three copolymers, only the P1 does not shown the minimum surface pressure during expansion, probably because a relative stable self-assembly structure is formed due to sequence and PMMA block and we will discuss that in the following morphology analysis.

3.3. Surface Morphologies of Langmuir-Blodgett Films. To characterize the interfacial aggregating behavior and thin film morphology formed in region 3, series of LB solid films are obtained using deposition on the glass substrate. AFM images of all three thin LB films are shown in Figure 5. 


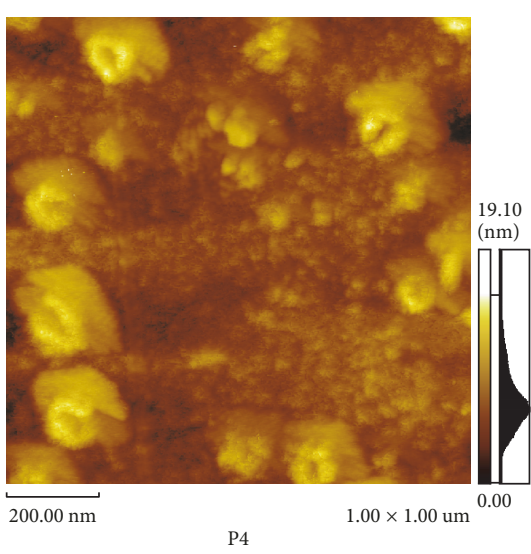

(a)

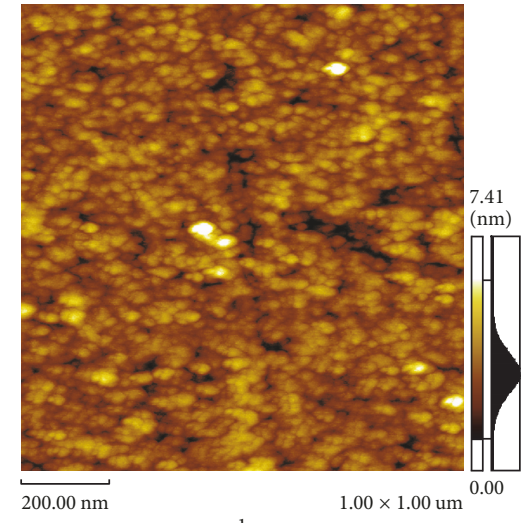

(b)

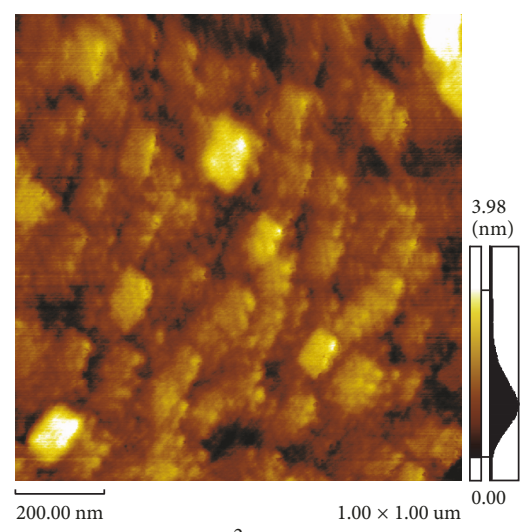

(c)

Figure 5: AFM images of thin film observed by P1 (a), P2 (b), and P3 (c) in region 3.

Figure 5(a) shows the morphology of LB film P1 formed. The micelles with volcano like structure are observed. The average thickness of the film is about $17.2 \mathrm{~nm}$. Correspondingly, the films prepared by $\mathrm{P} 2$ and $\mathrm{P} 3$ are, respectively, shown in Figures 4(b) and 4(c). The morphology of two LB films was completely different with P1. The light areas consisted of PTFEMA blocks which cannot form micelle or other regular arrangement but constitute flaky texture. The average thicknesses of those two films are $7.4 \mathrm{~nm}$ and $3.9 \mathrm{~nm}$, respectively. The great difference of thin film morphology and thickness is contributed to the different self-assembly behavior due to difference of block copolymer sequence. Based on the discussion of interfacial rheology, the lowest elasticity of P1 could easily be explained by the combination of AFM images. A core-corona shape 2D micelles is clearly detected from the AFM of $\mathrm{P} 1$ thin film; it probably formed due to the self-assembly during compression. Since both POSS and PTFEMA exhibit lower compressibility, the corona is formed by thick loop and tail structure to desorption of ester groups of PMMA as well as the higher compressibility of POSS and PTFEMA during compression. The light area in the images represents loop and tail structures of PTFEMA blocks, while compact PMMA blocks adsorbed on the surface form majority of dark area. Besides, when PMMA lies between POSS and PTFEMA blocks (P2 \& P3), the possibility of loop and tail structures formation is decreased, which is attributed to the lower aggregation behavior between POSS and PTFEMA blocks. While the PMMA block lies as the terminal group, the compressibility of Langmuir films is mainly contributed to the formation of compact PMMA aggregation in region 3 .

In order to further confirm the morphology of the three LB thin films, the surface elemental compositions of bulk material and the thin film are, respectively, analyzed by XPS as shown in Table 2. It is reasonable paying attention to Fluorine-to-Carbon $(\mathrm{F} / \mathrm{C})$ ratio which reflects the PTFEMA morphology at surface. Compared with the bulk material, a decrease of the $\mathrm{F} / \mathrm{C}$ ratio is detected on LB film formed by $\mathrm{P} 1$, while increase of $\mathrm{F} / \mathrm{C}$ ratio is observed by $\mathrm{P} 2$ and $\mathrm{P} 3$. It can be
TABLE 2: Surface elemental composition of three copolymers characterized by XPS.

\begin{tabular}{lccccc}
\hline Copolymer & Sample & C\% & O\% & F\% & $F / C$ \\
\hline \multirow{2}{*}{ P1 } & Bulk & 50.40 & 24.80 & 17.60 & 0.35 \\
& LB film & 38.77 & 41.20 & 8.43 & 0.22 \\
P2 & Bulk & 52.40 & 14.70 & 14.70 & 0.28 \\
& LB film & 36.14 & 33.93 & 11.31 & 0.31 \\
P3 & Bulk & 53.40 & 26.90 & 12.80 & 0.24 \\
& LB film & 34.74 & 32.86 & 11.70 & 0.34 \\
\hline
\end{tabular}

explained by the different migration behavior of fluorine alkyl group during compressing process. On the other hand, the detection thickness of surface XPS is around 1 to $3 \mathrm{~nm}$, which will only represent the top layer composition of the thin film. For the thin film formed by P1, the average film thickness is $17.2 \mathrm{~nm}$, higher than XPS detection depth, so the surface composition is much influenced by coating effect, causing the obviously decrease of $\mathrm{F} / \mathrm{C}$ ratio. It is quite possible that more PTFEMA chains are enshrouded and cannot be checked out due to the loop structure. In detail, the F/C ratio of the films is supposed to higher than the bulk materials. In the case of thin films formed by P2 and P3, PTFEMA block are easier to be compressed upward to the air/water interface due to the hydrophobicity of fluorine alkyl group. Therefore the upright of PTFEMA chain on the surface and the migration of fluoride to surface play a major role, increasing $\mathrm{F} / \mathrm{C}$ ratio.

Based on the arguments from interfacial rheology and surface morphology, a qualitative surface morphology model of thin films formed by different copolymers is shown in Figure 6(a).

The PMMA domain (blue part) with minimum thickness is still adsorbed on the surface. The PTFEMA domain (red part) with higher thickness is almost absorbed from the surface and upward to air and exposed to air, forming loop and tail structures. The POSS particles (black part) are partly coated in the PTFEMA chain. Meanwhile, the micelles 

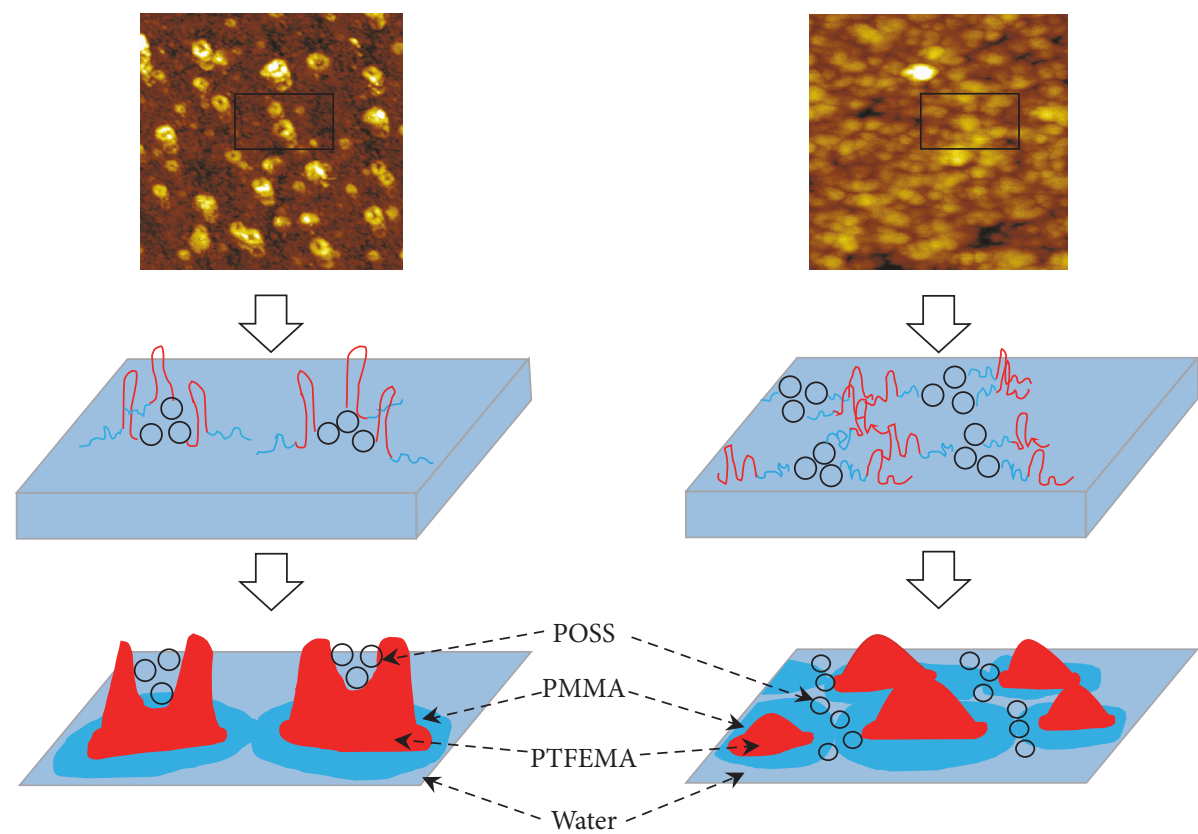

(a)
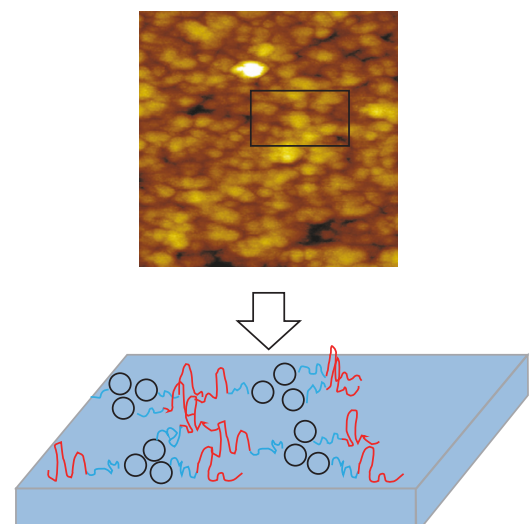

(b)

FIGURE 6: Qualitative surface morphology model of thin films formed by (a) P1, (b) P2, and P3 in high concentration at air/water interface.

showed a cave with certain depth in center of these cores, forming what we call crater-shaped structure.

Figure 6(b) showed the qualitative model of $\mathrm{P} 2$ formed $\mathrm{LB}$ film, the red PTFEMA chain only compressing to a relatively low level, and the blue PMMA chain is still absorbed on the subphase; the POSS particles are distributed around, coated in, or covered on the PTFEMA chain. P3 formed monolayer was similar as P2 and we just use the same model. The conclusion is that P2 is more likely to form thicker and sharper morphology of film like needle felting at region 3 while $\mathrm{P} 3$ tends to form lower and gentle terrains like mastoid.

3.4. The Interfacial Self-Assembly Structure Evolution. From the $\pi$-MMA isotherms, hysteresis cycles, AFM images, and XPS analysis, we could propose the mechanism of the two dimensional aggregation of all three tadpole-shaped POSSbased copolymers at air/water interface, shown in Figure 7.

At the initial stage of compression (region 1), all three copolymers freely stretch at the air/water interface and P1 is taken as representative. Both PMMA and PTFEMA blocks are adsorbed on the interface and the thickness of Langmuir film is limited. All three copolymers present free conformations change in two dimension in region 1 . As compressing in region 2, the molecules begin to touch with each other. PTFEMA and PMMA blocks are, respectively, aggregated to adapt to the decrease of surface area. The aggregation behavior of P1 formed film in this region is shown in Figure 6 . The quasi-2D micelles were initially formed, which showed the core-corona micelles with the POSS core and looped PTFEMA and PMMA as corona. For P2 and P3, although the PTFEMA blocks aggregated upward to the air, but with restriction to the location of PTFEMA and PTFEMA blocks, the POSS core was only distributed around the PMMA aggregation and quasi-2D micelle cannot be formed.

With the deeper compression process, copolymer concentration at air/water interface was increased. The morphology of P1 formed film was shown in Figure 6(a). In this region, the desorption of ester groups from PTFEMA occurred; most of PTFEMA blocks were exposed to air to form loop and tail structures with further compressing. Much thicker film with specific quasi-2D micelles is further obtained. Besides, the morphology of P2 and P3 formed films is, respectively, shown in Figures 6(b) and 6(c). Obviously there's no any micelle formed, but only the flaky texture without regular pattern is shown. The thickness of loop and tail structures caused by further compressing is much lower than P1. As the thickness of film mainly depended on PMMA ratio; P2 formed film is thicker than P3. In brief, the intermolecular force on the interface plays a great role in thin film structure. A weak intramolecular repulsion is determined for P1 and the thin film could form 2D micelle structure due to the low elasticity. And thin film of P3 showed flaky aggregates mainly due to the higher intermolecular repulsive force.

\section{Conclusion}

In this work, the air/water interfacial self-assembly of tadpole-shaped fluorine containing POSS-based copolymers with different sequence and block ratio was carried out by Langmuir technique. Based on study of the interfacial rheology, the relationship between compressibility of the thin film and molecular structure of the block copolymer is illustrated. While two blocks are shown in comparable length, the compressibility was higher while POSS and 

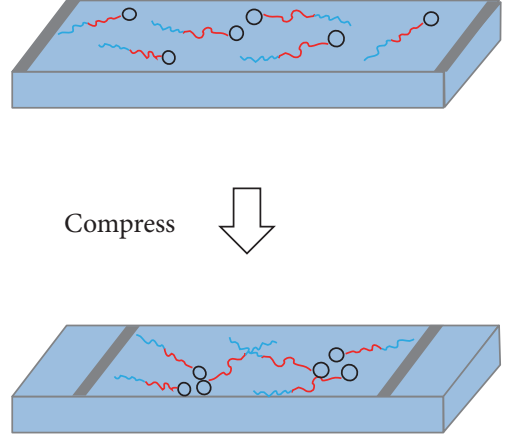

Compress

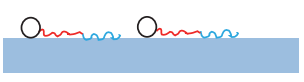

Region 1

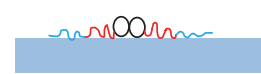

Region 2

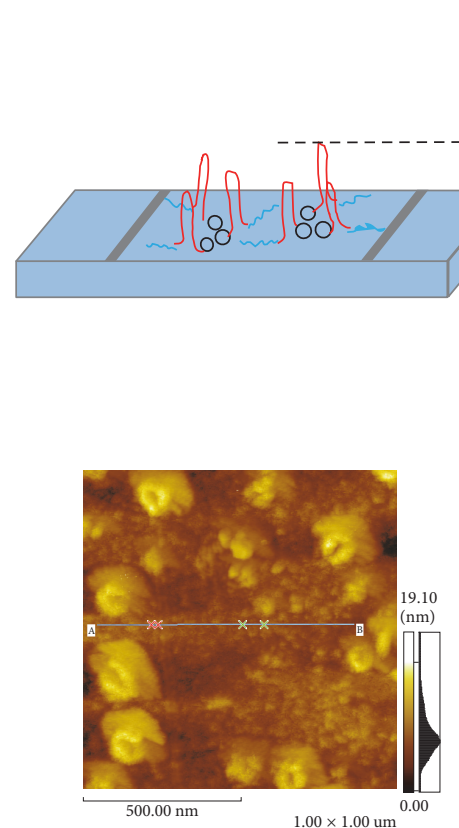

(a)

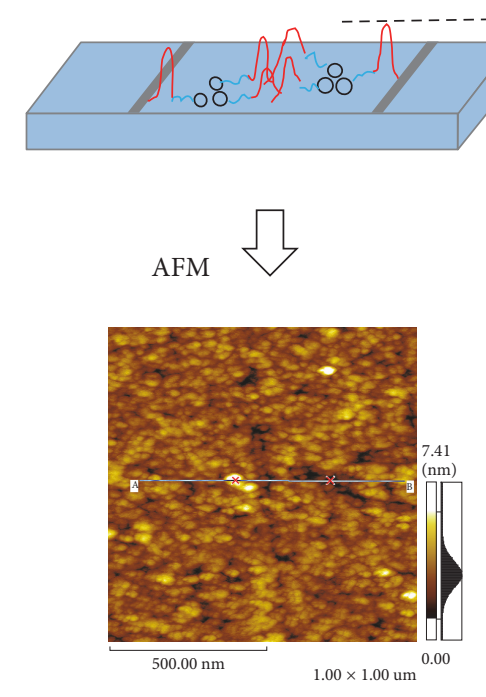

(b)
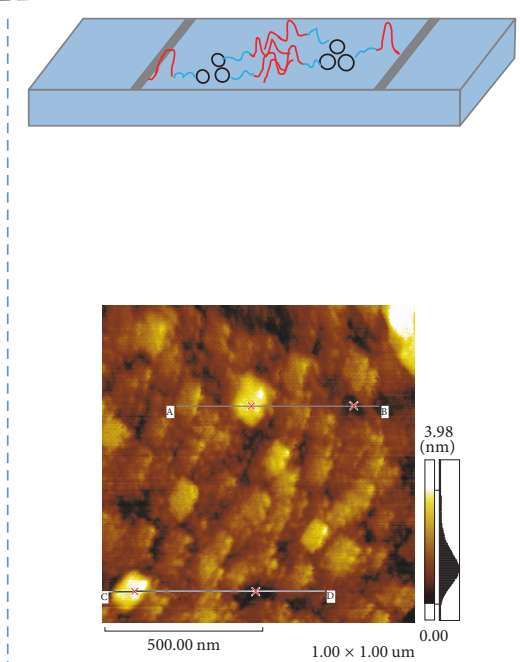

(c)

O POSS

$\sim$ PTFEMA

$\sim$ PMMA

Water

FIGURE 7: Mechanism for the formational changes of copolymer POSS-PTFEMA- $b$-PMMA at air/water interface during compressing process.

PTFEMA were connected to each other. However, P2 and P3 both showed higher elasticity because of larger repulsion of terminal PTFEMA blocks and P3 presented higher compressibility attribute to entanglement between nearby PMMA blocks. The formation of quasi-2D micelle with core-corona during compression of P1 was proved by analyzing the AFM images and XPS. Accordingly, copolymers POSS-PMMA $277^{-}$ $b$-PTFEMA 130 and POSS-PMMA $466-b$-PTFEMA 172 could form gentle terrains like mastoid. The thickness and diameter of mastoid were greatly related to PMMA block ratio as well.

\section{Conflicts of Interest}

The authors declare no potential conflicts of interest.

\section{Acknowledgments}

This research is supported by Natural Science Foundation of Shaanxi Province (no. 2016JM2023) and Natural Science Foundation of Shenzhen (JCYJ20170306161101003).

\section{Supplementary Materials}

Figure S1. Synthesis route of POSS-PTFEMA-b-PMMA. Figure S2. 1H NMR spectra of POSS-Cl and all three tadpoleshaped copolymers: (a) POSS-Cl, (b) POSS-PTFEMA-bPMMA, (c) POSS-PMMA1-b-PTFEMA, and (d) POSSPMMA2-b-PTFEMA. Table S1. Characteristics of all three copolymers. (Supplementary Materials) 


\section{References}

[1] K. Pielichowski, J. Njuguna, B. Janowski, and J. Pielichowski, "Polyhedral oligomeric silsesquioxanes (POSS)-containing nanohybrid polymers," in Supramolecular Polymers Polymeric Betains Oligomers, vol. 201 of Advances in Polymer Science, pp. 225-296, Springer, Berlin, Germany, 2006.

[2] D. Gnanasekaran, K. Madhavpan, and R. S. R. Reddy, "Developments of polyhedral oligomeric silsesquioxanes (POSS), POSS nanocomposites and their applications: a review," Journal of Scientific and Industrial Research, vol. 68, no. 6, pp. 437-464, 2009.

[3] E. Ayandele, B. Sarkar, and P. Alexandridis, "Polyhedral Oligomeric Silsesquioxane (POSS)-containing polymer nanocomposites," Nanomaterials, vol. 2, no. 4, pp. 445-475, 2012.

[4] X. T. Cao, A. M. Showkat, L. G. Bach et al., "Synthesis and characterization of photoluminescent hybrids of poly $(\varepsilon-$ caprolactone)-grafted-polyhedral oligosilsesquioxane by using a combination of ring-opening polymerization and click chemistry," Journal of the Korean Physical Society, vol. 66, no. 1, pp. 108-112, 2015.

[5] S. Li, Y. Liu, S. Ji, Z. Zhou, and Q. Li, "Synthesis and selfassembly behavior of thermoresponsive poly(oligo(ethylene glycol) methyl ether methacrylate)-POSS with tunable lower critical solution temperature," Colloid and Polymer Science, vol. 292, no. 11, pp. 2993-3001, 2014.

[6] H. Hou, Y. Gan, J. Yin, and X. Jiang, "Multifunctional POSSbased nano-photo-initiator for overcoming the oxygen inhibition of photo-polymerization and for creating self-wrinkled patterns," Advanced Materials Interfaces, vol. 1, no. 9, Article ID 1400385, 2014.

[7] Y. S. Seo, H. Yu, J. Kim, and S. Park, Abstr Pap Am Chem S, Thinfilm behavior of polystyrene-block-poly(methyl methacrylate) diblock copolymer at the air-water interface. Abstr Pap Am Chem S 21, U419-U419, 1999.

[8] X. Qiang, X. Ma, Z. Li, and X. Hou, "Synthesis of star-shaped polyhedral oligomeric silsesquioxane (POSS) fluorinated acrylates for hydrophobic honeycomb porous film application," Colloid and Polymer Science, vol. 292, no. 7, pp. 1531-1544, 2014.

[9] J. Miao, L. Cui, H. P. Lau, P. T. Mather, and L. Zhu, "Self-assembly and chain-folding in hybrid coil-coil-cube triblock oligomers of polyethylene-b-poly(ethylene oxide)-bpolyhedral oligomeric silsesquioxane," Macromolecules, vol. 40, no. 15, pp. 5460-5470, 2007.

[10] K. Koh, S. Sugiyama, T. Morinaga et al., "Precision synthesis of a fluorinated polyhedral oligomeric silsesquioxane-terminated polymer and surface characterization of its blend film with poly(methyl methacrylate)," Macromolecules, vol. 38, no. 4, pp. 1264-1270, 2005.

[11] A. C. Kucuk, J. Matsui, and T. Miyashita, "Erratum: Effects of hydrogen bonding on the monolayer properties of amphiphilic double-decker-shaped polyhedral silsesquioxanes (Langmuir (2011) 27 (6381) DOI:10.1021/la200604w)," Langmuir, vol. 27, no. 14, p. 9068, 2011.

[12] Y. Seo, S. G. Youm, J. H. Kim et al., "Aggregation behavior of a polystyrene-b-poly(phenylsilsesquioxane) H-type copolymer at the air/water interface," Polymer Journal, vol. 53, no. 11, pp. 2223-2232, 2012.

[13] A. Wamke, K. Dopierała, K. Prochaska, H. Maciejewski, A. Biadasz, and A. Dudkowiak, "Characterization of Langmuir monolayer, Langmuir-Blodgett and Langmuir-Schaefer films formed by POSS compounds," Colloids and Surfaces A: Physicochemical and Engineering Aspects, vol. 464, pp. 110-120, 2015.
[14] B. Jiang, W. Tao, X. Lu et al., "A POSS-based supramolecular amphiphile and its hierarchical self-assembly behaviors," Macromolecular Rapid Communications, vol. 33, no. 9, pp. 767772, 2012.

[15] R. Gunawidjaja, F. Huang, M. Gumenna et al., "Bulk and surface assembly of branched amphiphilic polyhedral oligomer silsesquioxane compounds," Langmuir, vol. 25, no. 2, pp. 11961209, 2009.

[16] Z. Zhang, L. Hong, J. Li et al., "One-pot synthesis of well-defined amphiphilic alternating copolymer brushes based on POSS and their self-assembly in aqueous solution," RSC Advances, vol. 5, no. 28, pp. 21580-21587, 2015.

[17] W. T. E. Bosker, M. A. Cohen Stuart, and W. Norde, "(Quasi) $2 \mathrm{D}$ aggregation of polystyrene-b-Dextran at the air-water interface," Langmuir, vol. 29, no. 8, pp. 2667-2675, 2013.

[18] Y.-S. Seo, F. Ahmad, K. Shin et al., "Interfacial behavior of randomly charged sulfonated polystyrene (PSS) at the air/water interface," Colloids and Surfaces A: Physicochemical and Engineering Aspects, vol. 313-314, pp. 660-665, 2008.

[19] I. I. Perepichka, A. Badia, and C. G. Bazuin, "Nanostrand formation of block copolymers at the air/water interface," ACS Nano, vol. 4, no. 11, pp. 6825-6835, 2010.

[20] T. J. Joncheray, K. M. Denoncourt, M. A. R. Meier, U. S. Schubert, and R. S. Duran, "Two-dimensional self-assembly of linear polyethylene oxide-b-poly( $\varepsilon$ - caprolactone) copolymers at the air-water interface," Langmuir, vol. 23, no. 5, pp. $2423-$ 2429, 2007.

[21] Y.-S. Seo, K.-S. Kim, K. Shin et al., "Morphology of amphiphilic gold/dendrimer nanocomposite monolayers," Langmuir, vol. 18, no. 15, pp. 5927-5932, 2002.

[22] Y. Seo, C. Y. Cho, M. Hwangbo, H. J. Choi, and S. M. Hong, "Effect of temperature on the interfacial behavior of a polystyrene-b- poly(methyl methacrylate) diblock copolymer at the air/water interface," Langmuir, vol. 24, no. 6, pp. 23812386, 2008.

[23] H. Tanaka, Y. Kunai, N. Sato, and T. Matsuyama, "Effect of the graft chain length and density on the morphology of radiationmodified polysilane monolayers at the air/water interface," Journal of Colloid and Interface Science, vol. 363, no. 2, pp. 440445, 2011.

[24] I. I. Perepichka, Q. Lu, A. Badia, and C. G. Bazuin, "Understanding and controlling morphology formation in LangmuirBlodgett block copolymer films using PS-P4VP and PSP4VP/PDP," Langmuir, vol. 29, no. 14, pp. 4502-4519, 2013.

[25] Q. Lu and C. G. Bazuin, "Solvent-assisted formation of nanostrand networks from supramolecular diblock copolymer/ surfactant complexes at the air/water interface," Nano Letters, vol. 5, no. 7, pp. 1309-1314, 2005.

[26] I. I. Perepichka, K. Borozenko, A. Badia, and C. G. Bazuin, "Pressure-induced order transition in nanodot-forming diblock copolymers at the air/water interface," Journal of the American Chemical Society, vol. 133, no. 49, pp. 19702-19705, 2011.

[27] V. Ferri, M. Elbing, G. Pace et al., "Light-powered electrical switch based on cargo-lifting azobenzene monolayers," Angewandte Chemie International Edition, vol. 47, no. 18, pp. 34073409, 2008.

[28] J. M. Mativetsky, G. Pace, M. Elbing, M. A. Rampi, M. Mayor, and P. Samorì, "Azobenzenes as light-controlled molecular electronic switches in nanoscale metal-molecule-metal junctions," Journal of the American Chemical Society, vol. 130, no. 29, pp. 9192-9193, 2008. 
[29] Y. Seo, A. R. Esker, D. Sohn, H.-J. Kim, S. Park, and H. Yu, "Study on the behaviors of different polystyrene-block-poly(methyl methacrylate) diblock copolymers adsorbed at the air/water interface," Langmuir, vol. 19, no. 8, pp. 3313-3322, 2003.

[30] G. Li Destri, F. Miano, and G. Marletta, "Structure-rheology relationship in weakly amphiphilic block copolymer langmuir monolayers," Langmuir, vol. 30, no. 12, pp. 3345-3353, 2014.

[31] G. Liu, S. Yang, and G. Zhang, "Conformational changes of poly(N-isopropylacrylamide) chains at air/water interface: Effects of temperature, compression rate, and packing density," The Journal of Physical Chemistry B, vol. 111, no. 14, pp. 36333639, 2007.

[32] A. Brzozowska, J. Paczesny, P. Parzuchowski et al., "Hyperbranched polyesters terminated with alkyl chains of different length at the air/water interface and on solid substrates," Macromolecules, vol. 47, no. 15, pp. 5256-5268, 2014.

[33] Y. Seo, J.-H. Im, J.-S. Lee, and J.-H. Kim, "Aggregation behaviors of a polystyrene-b-poly(methyl methacrylate) diblock copolymer at the air/water interface," Macromolecules, vol. 34, no. 14, pp. 4842-4851, 2001.

[34] Y. Seo, K. Paeng, and S. Park, "Molecular weight effect on the behaviors of polystyrene-block-poly(methyl methacrylate) diblock copolymers at air/water interface," Macromolecules, vol. 34, no. 25, pp. 8735-8744, 2001.

[35] Y. Chen, T. Liu, G. Xu et al., "Aggregation behavior of X-shaped branched block copolymers at the air/water interface: effect of block sequence and temperature," Colloid and Polymer Science, vol. 293, no. 1, pp. 97-107, 2014.

[36] R. R. Thomas, D. R. Anton, W. F. Graham et al., "Preparation and surface properties of acrylic polymers containing fluorinated monomers," Macromolecules , vol. 30, no. 10, pp. 28832890, 1997.

[37] B. B. Akhremitchev, B. K. Mohney, K. G. Marra, T. M. Chapman, and G. C. Walker, "Atomic force microscopy studies of hydration of fluorinated amide/urethane copolymer film surfaces," Langmuir, vol. 14, no. 15, pp. 3976-3982, 1998.

[38] D. Han, L. Zhu, Y. Chen, W. Li, X. Wang, and L. Ning, "Synthesis of fluorosilicone monomer and application in hydrophobic surface of acrylic copolymer," Journal of Applied Polymer Science, vol. 132, no. 18, Article ID 41926, 2015.

[39] H. Zou, S. Lin, Y. Tu et al., "Simple approach towards fabrication of highly durable and robust superhydrophobic cotton fabric from functional diblock copolymer," Journal of Materials Chemistry A, vol. 1, no. 37, pp. 11246-11260, 2013.

[40] G. Li, A. Xu, B. Geng, S. Yang, G. Wu, and S. Zhang, "Synthesis and characterization of fluorinated diblock copolymer of 2,2,2trifluoroethyl methacrylate and methyl methacrylate based on RAFT polymerzation," Journal of Fluorine Chemistry, vol. 165, pp. 132-137, 2014.

[41] Y. Zhang, Y. Wang, H. Zhang et al., "Synthesis and surface-active behavior of new fluorinated hyperbranched polymer," Journal of Surfactants and Detergents, vol. 17, no. 5, pp. 967-975, 2014.

[42] H. Zhu, J. Matsui, S. Yamamoto, T. Miyashita, and M. Mitsuishi, "Solvent-dependent properties of poly(vinylidene fluoride) monolayers at the air-water interface," Soft Matter, vol. 11, no. 10, pp. 1962-1972, 2015.

[43] A. Qu, X. Wen, P. Pi, J. Cheng, and Z. Yang, "Gradient distribution of fluorine on the film surface of the organic-inorganic hybrid fluoropolymer," Colloids and Surfaces A: Physicochemical and Engineering Aspects, vol. 345, no. 1-3, pp. 18-25, 2009.
[44] B. J. Privett, J. Youn, S. A. Hong et al., "Antibacterial fluorinated silica colloid superhydrophobic surfaces," Langmuir, vol. 27, no. 15, pp. 9597-9601, 2011.

[45] A. Stoffers, C. Oberdorfer, and G. Schmitz, "Controlled field evaporation of fluorinated self-assembled monolayers," Langmuir, vol. 28, no. 1, pp. 56-59, 2012.

[46] R. B. Cheyne and M. G. Moffitt, "Controllable organization of quantum dots into mesoscale wires and cables via interfacial block copolymer self-assembly," Macromolecules , vol. 40, no. 6, pp. 2046-2057, 2007.

[47] H. Li, R. Sachsenhofer, W. H. Binder et al., "Hierarchical organization of poly(ethylene oxide)-block-poly(isobutylene) and hydrophobically modified fe2o3 nanoparticles at the air/water interface and on solid supports," Langmuir, vol. 25, no. 14, pp. 8320-8329, 2009.

[48] S. S. Lamarre, C. Lemay, C. Labrecque, and A. M. Ritcey, "Controlled 2D organization of gold nanoparticles in block copolymer monolayers," Langmuir, vol. 29, no. 34, pp. 1089110898, 2013.

[49] S. S. Lamarre, H. Yockell-Lelièvre, and A. M. Ritcey, "Assembly of polystyrene-coated gold nanoparticles at the air-water interface," Journal of Colloid and Interface Science, vol. 443, pp. 131s136, 2015.

[50] R. B. Cheyne and M. G. Moffitt, "Hierarchical nanoparticle/block copolymer surface features via synergistic selfassembly at the air - Water interface," Langmuir, vol. 21, no. 23, pp. 10297-10300, 2005.

[51] B. P. Gagnon and M.-V. Meli, "Effects on the self-assembly of $\mathrm{n}$-Alkane/gold nanoparticle mixtures spread at the air-water interface," Langmuir, vol. 30, no. 1, pp. 179-185, 2014.

[52] A. Maestro, H. M. Hilles, F. Ortega, R. G. Rubio, D. Langevin, and F. Monroy, "Reptation in langmuir polymer monolayers," Soft Matter, vol. 6, no. 18, pp. 4407-4412, 2010.

[53] A. Maestro, F. Ortega, R. G. Rubio, M. A. Rubio, J. Kärgel, and R. Miller, "Rheology of poly(methyl methacrylate) Langmuir monolayers: Percolation transition to a soft glasslike system," The Journal of Chemical Physics, vol. 134, no. 10, Article ID 104704, 2011.

[54] L. Dominique and M. Francisco, "Interfacial rheology of polyelectrolytes and polymer monolayers at the airwater interface," Current Opinion in Colloid \& Interface Science, vol. 15, pp. 283293, 2010. 

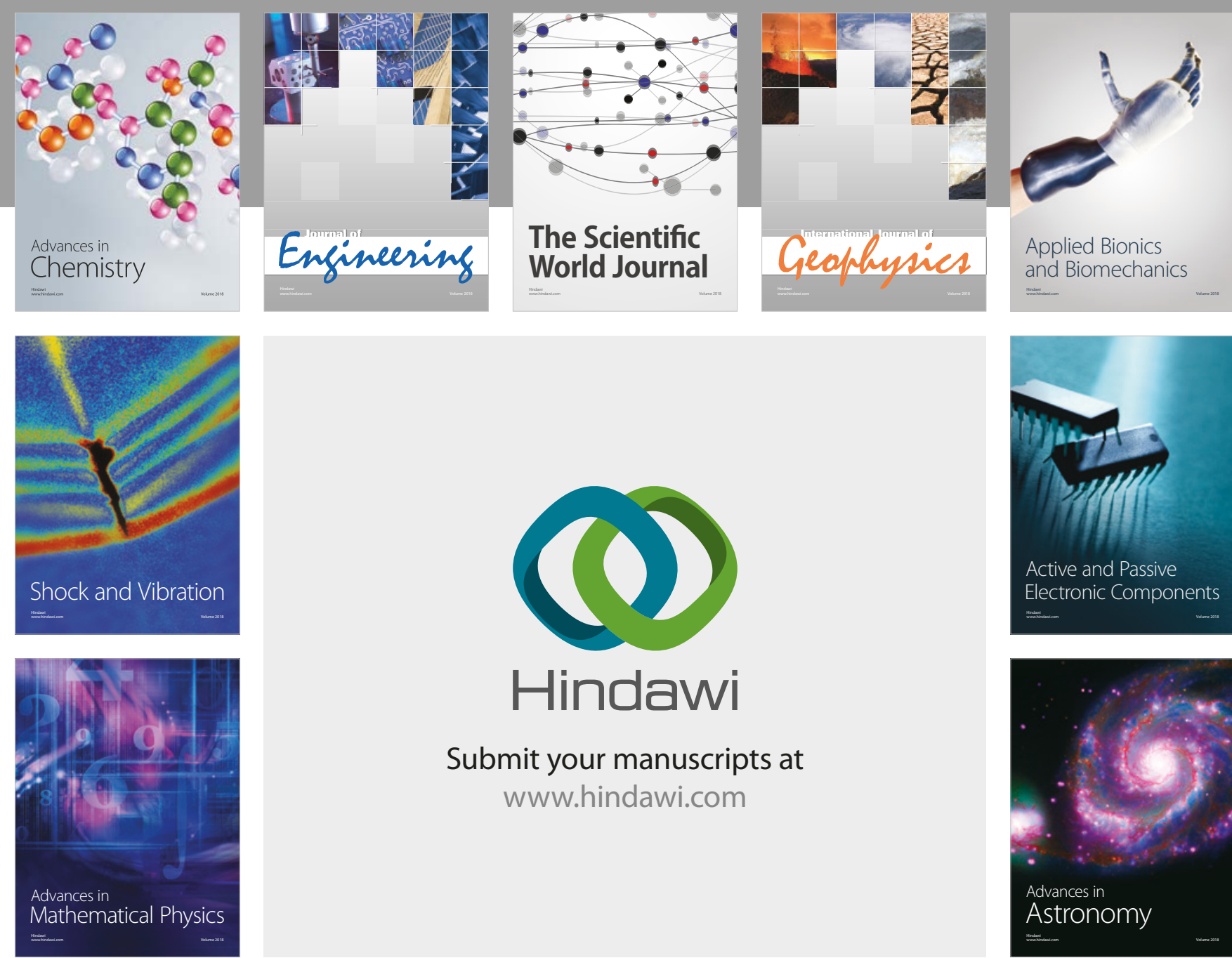

Submit your manuscripts at

www.hindawi.com

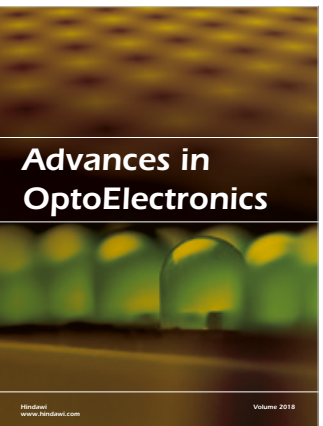

\section{Rotcting Machinery}
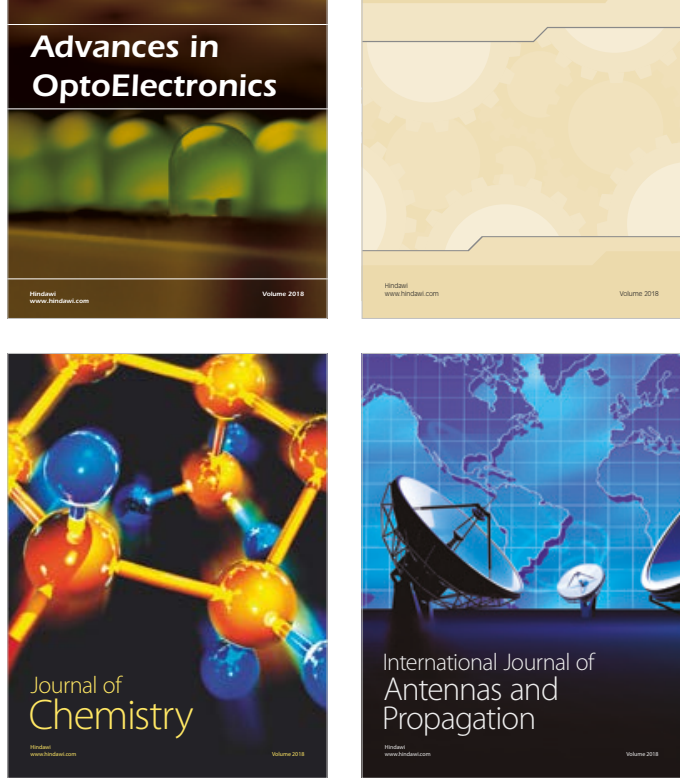

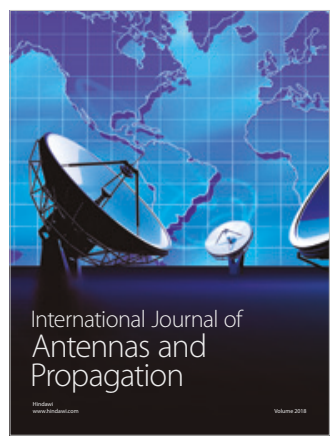

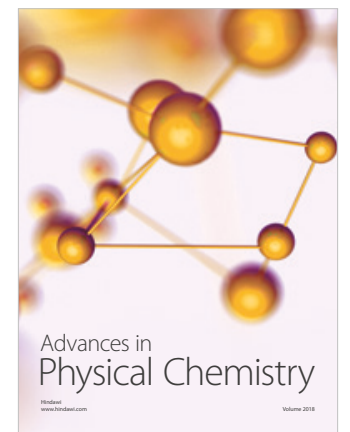

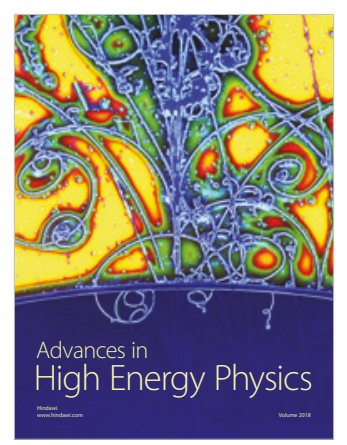

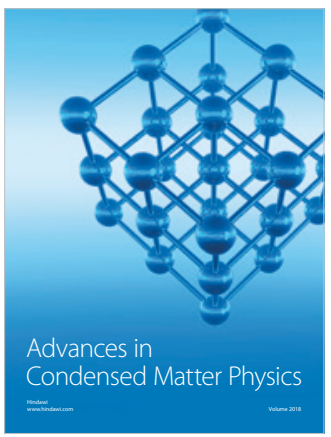

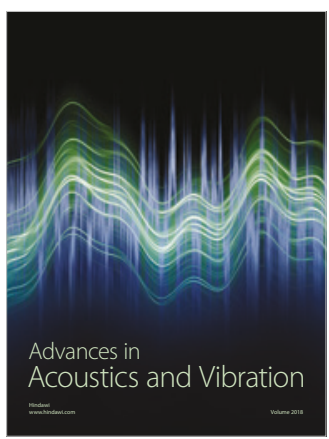

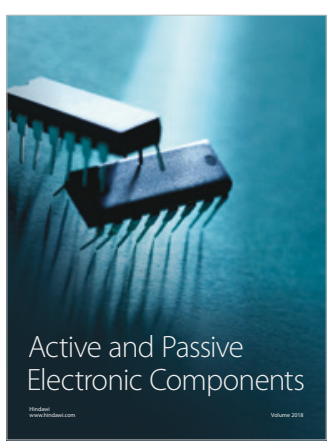
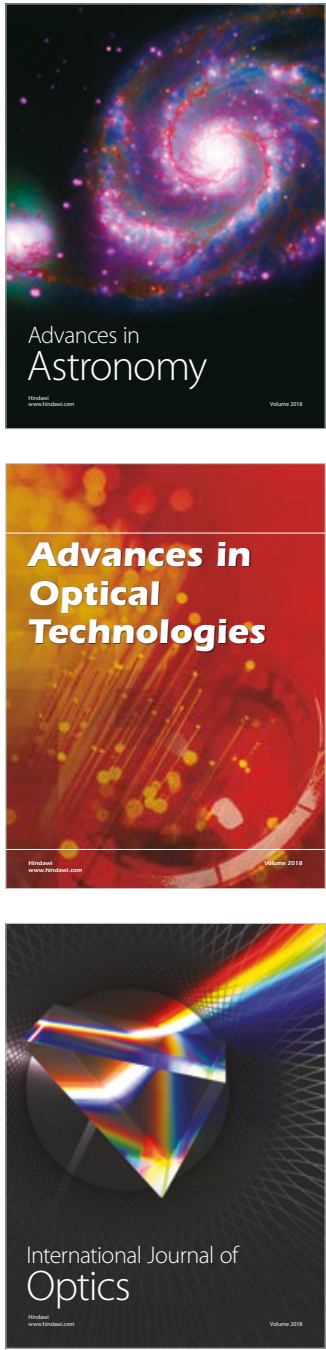